\title{
La gestión de la información en la administración pública desde la perspectiva de la recuperación de la información
}

\author{
Information management in public administrations in the context of information retrieval
}

\author{
María del Carmen CRUz GIL \\ mariadelcarmencruzgil@gmail.com
}

\begin{abstract}
Resumen
Se reflexiona sobre la relevancia de la gestión de la información en la administración pública, enfatizando el uso de normativas y técnicas documentales para asegurar la recuperación de la información pública. En particular, se insiste en la importancia de mapear y medir los flujos de información, implementar esquemas de metadatos y herramientas de organización del conocimiento y desarrollar políticas de alfabetización informacional.
\end{abstract}

Palabras clave: Administración pública. Recuperación de la información. Gestión de la información. Metadatos. Alfabetización informacional.

\section{Introducción}

La necesidad de información no es una necesidad primaria, sino una necesidad secundaria que surge de las necesidades de carácter más básico (Wilson, 1999, p. 252). Por tanto, la gestión de la información, disciplina ya de por sí compleja, se agrava por su alto nivel de generalización en el contexto de las Organizaciones (García, 2008, p. 9). Como señala Ponjuán (2007, p. 23),

\begin{abstract}
La Organización que quiera tener una gestión de la información, se ve obligada a tener un dominio de los diferentes tipos de informaciones que se manejan, de la dinámica de sus flujos, del ciclo de vida de cada información y de la cultura informacional del personal.
\end{abstract}

La extracción de esta información es uno de los medios para lograr ventajas competitivas (Ruthven, 2008, p. 43), por lo que como dice López (2008, p. 5; citado en Costa, Silva y Ramalho, 2010 , p. 131), no se puede descuidar que los archivos están presentes en todas las esferas de la sociedad, mediante el tratamiento de los documentos como productos múltiples de actividades de la organización y gestión humana.

Ahora bien, la información en las organizaciones obedece a un flujo; por lo que su mapeo es fundamental. Por workflow se entiende un flujo de trabajo en el que que intervienen documen-

\begin{abstract}
The relevance of information management for public administrations is discussed, with an emphasis in ensuring that public information is properly retrieved and used. Information flow analysis, information literacy, and knowledge organization and metadata schemas are recommended as particularly relevant tools.
\end{abstract}

Keywords: Public administration. Information retrieval. Information management. Metadata. Information literacy.

tos, información y tareas que realizan los usuarios con la finalidad de concluir un proceso que, a su vez forma parte de un objetivo más general (Solana González, 2007, p. 1)—. Por tanto, que el proceso de workflow quede registrado va a permitir a la organización un análisis exhaustivo de la información que gestiona para la toma de decisiones, además de contribuir a aumentar la eficacia y eficiencia de sus procesos. La dinámica y el control de flujos va a permitir conocer entre otros: a) los tipos y volúmenes de información que fluyen en el sistema y en cada subsistema, además de sus características; b) el tiempo empleado en la obtención de la información; c) las distorsiones y el papel que desempeña cada departamento; y d) el almacenamiento de datos transitorios y permanentes en la organización.

Ponjuan (2007, p. 31) advierte que el análisis de estos flujos debe hacerse cada cierto tiempo y que ante la entrada de nuevas personas o cambios estructurales debe hacerse de manera inmediata todavía más profunda.

Miguel Castaño (apud Muñoz Cañavate, 2003, p. 35), en su momento, ha sostenido que uno de los problemas originados en la administración pública, quizás uno de los principales, es la cantidad ingente de datos, la desestructuración de la información y la dispersión de las fuentes, 
merced de una burocracia que crece sin límites, como fácilmente se comprueba en la práctica. Problemas que se han intentado solucionar con remedios estrictamente informáticos sin tener en cuenta los flujos informacionales ni los fines o la misión y los valores de la organización. También Muñoz Cañavate (2003, p. 36) manifiesta la inexistencia de unas prácticas tendentes a mostrar el acceso a la información como una parte natural del sistema administrativo y, como consecuencia, la falta también de accesibilidad. Dicha inadecuación entre información e informática puede ser motivo real del éxito o fracaso en la implementación de sistemas de información en las organizaciones, por lo que se considera necesario averiguar las posibles soluciones.

\section{Objetivos y metodología}

Ya en los resultados de un estudio de investigación previo (Cruz Gil, 2012), se detectaron numerosos factores que inhibían la recuperación de la información en la Administración Pública española. Por ello, sería necesario, dado el vacío de la investigación, un estudio más amplio para conocer el porqué de resultados tan poco favorables en el contexto de la recuperación de la información en la Administración Pública como aquellos que se han encontrado. Por otra parte, Martín-Pozuelo (2012, p. 13) ha llamado también la atención sobre la escasez de estudios sobre el tema.

Así, pues parece conveniente realizar una investigación desde la que proponer distintas soluciones al problema detectado. Sus principales objetivos generales son tres: conocer la importancia de la recuperación de la información en la Administración Pública española; conocer la importancia de la relación entre la recuperación de la información en la Administración Pública española con las Ciencias de la Documentación; y aportar un conjunto de pautas o recomendaciones generales. Específicamente, se persigue que los factores inhibidores de la recuperación de la información en la Administración Pública disminuyan.

Desde el punto de vista metodológico, el estudio parte de un estudio de la teoría de la recuperación de la información desde el punto de vista de las Ciencias de la Documentación, por un lado, y de la gestión de la información en la administración pública con énfasis en la recuperación de la información, por el otro.

\section{Resultados}

A continuación se resalta un conjunto de ideas clave sobre la gestión de la información, el intercambio de información y la necesidad de implantar normativas y técnicas documentales en la Administración Pública, enfatizando en particular el uso de esquemas de metadatos y la alfabetización informacional.

\subsection{La gestión de la información}

en la administración pública

Cornella Alfons (2000), citado en García (2010, p. 2), afirma "que las organizaciones y las personas se enfrentan a la necesidad de gestionar la información de manera eficiente", puesto que la desproporción entre el volumen creciente de información a la que se tiene acceso, por un lado, y la escasa disponibilidad de conocimiento, por el otro, implican que se exponga a las organizaciones e individuos a un mayor riesgo de caer en la parálisis informativa. Como consecuencia, advierte que las organizaciones que pretenden sobrevivir en la sociedad del conocimiento deberán incrementar su capital intelectual además de diseñar y aplicar nuevas estrategias de generación de conocimiento.

De igual modo, González Lorca (2006, p. 29) corrobora que la información adquiere un valor añadido, ya que su control y manejo es lo que va a determinar la competitividad de unas organizaciones sobre otras. En consecuencia, es vital establecer procedimientos que garanticen su tratamiento, almacenamiento y difusión, asegurando o aumentando así su eficacia y eficiencia en la organización.

Los documentos son todavía la base de toda la actividad organizativa. En ellos se reflejan las acciones de la organización, y se refleja el conocimiento de la organización, por lo que deben ser tratados de forma adecuada para que la Administración Pública pueda ofrecer el servicio necesario a sus ciudadanos. Esto lleva implícito establecer procedimientos en materia de gestión documental (1) que permitan la integración de la documentación y la información en los sistemas de la organización (figura 1).

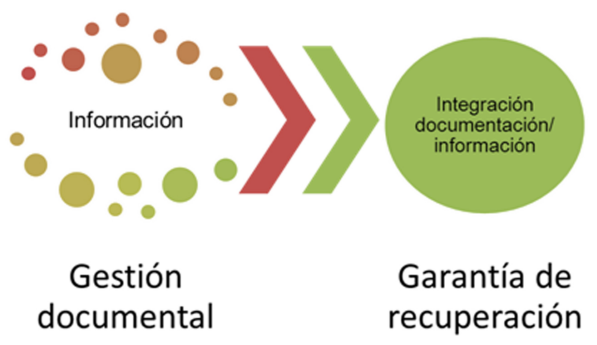

Figura 1. Procedimientos relevantes en materia de gestión documental en las organizaciones

García Alsina (2008, p. 10) plantea la aplicación pragmática de la normativa existente en las 
diferentes fases de la cadena documental, analizada en relación con el ciclo del conocimiento. De esta forma, la Administración podrá garantizar los derechos fundamentales de los ciudadanos y hacerlo además de modo proactivo, elevando su competitividad a través de su eficiencia y eficacia.

García Marco (2008, p. 13) expone que la información y la documentación en las empresas y administraciones públicas es un campo fronterizo de las Ciencias Documentales por dos razones. En primer lugar, son actividades informativas documentales en estrecha relación con la gestión de las organizaciones; en consecuencia, constituyen subespecialidades que contribuyen a nutrir a las Ciencias Documentales, en su conjunto, con los modelos y técnicas procedentes de ese campo. En segundo lugar, la gestión de la información en las organizaciones contribuye a extender el alcance y la utilidad de los modelos y técnicas documentales más allá de las fronteras de la educación, la investigación y la cultura.

\subsection{El intercambio de información}

en la administración pública

Se identifica a Albert Gore (1993), citado en Alcaide Muñoz (2012, p. 33), como la persona que utilizó por primera vez el término eGobierno haciendo referencia a la implementación de las Tecnologías de Información y Comunicación (TIC) en la Administración Pública. En definitiva, el e-gobierno conlleva el uso sistemático de herramientas que tienen el potencial de automatizar y distribuir en red los procesos internos y externos de las Administraciones Públicas, favoreciendo una presentación de servicios de modo fácil y eficiente. Según el Observatorio Europeo de Tecnología de la Información del 2002 (citado en Alcaide Muñoz, 2012, p. 34), este cambio otorga a la Administración Pública la oportunidad de revisar sus procesos y procedimientos y también a replantearse su modelo de funcionamiento para conseguir ser más eficaz y eficiente.

Estas razones han llevado a la Administración Pública a involucrase en proyectos orientados al intercambio de información, como el proyecto para el intercambio en las Administraciones Públicas INDALO, en el que entre sus dominios de actuación aborda la normalización de la gestión documental para garantizar la optimización de procesos y, por tanto, la obtención de beneficios (González Lorca, 2006, p. 40).

Bustelo Ruesta (2011, p. 130) también plantea la gestión de la información creada y recibida por una organización en el transcurso de sus actividades y el desarrollo de sus funciones como objetivo principal para la integración de procesos.

Muñoz Cañavate (2003, p. 71) comenta la existencia de proyectos para localizar rápidamente cualquier tipo de información administrativa y la puesta en marcha de sistemas para transferir al formato digital los documentos en papel. Hay otros, como el proyecto dirigido a desarrollar sistemas de información para las Administraciones, el Government Online, que tiene estructuradas unas líneas de trabajo entre las que se encuentra la reutilización de información administrativa, para evitar que diferentes departamentos que utilizan la misma información y datos comunes tengan la misma información en lugares distintos.

También la directiva europea 2003/98/CE para la reutilización de la información en el sector público plantea la conveniencia de la disponibilidad de los datos de carácter público elaborados o gestionados por las administraciones (Pastor Sánchez, 2014, p. 1). La aplicación de esta norma garantiza la capacidad de interconexión y la reutilización de la información, además de una mayor transparencia, que se ve reforzada también por la nueva directiva 2013/37/UE sobre reutilización de la información. Pastor Sánchez (2014, p. 3) ha indicado la imprescindible vinculación entre datos abiertos y gestión de la información, ya que es lo que va a permitir identificar los datos de los que es posible su publicación y, también, el modo en el que debe hacerse.

Por otro lado, la resolución del 3 de octubre de 2012 (BOE 31-10-2012) establece los estándares para alcanzar la interoperabilidad y los organiza en función de una cadena formada por los siguientes elementos (figura 2).

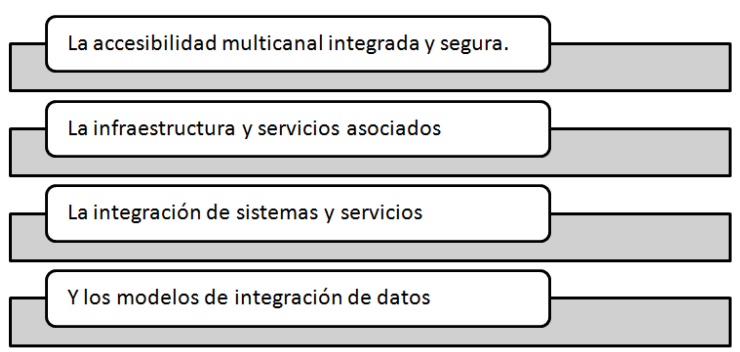

Figura 2. Elementos de la cadena de interoperabilidad (BOE 31-10-2012)

Además de la normativa respecto a la interoperabilidad, también se han ido produciendo otras recomendaciones. El Libro Verde de la información en el sector público, por ejemplo, publicado 
en 1998, indica ya que una de las funciones principales del gobierno electrónico es disponer de servicios orientados a ordenar y clasificar la información recogida, para tenerla a disposición de quien la solicite. En el momento de su elaboración se reconocía que la información en la Unión Europea se encontraba dispersa y que el reto para los Estados miembros era encontrar formas de tenerla más clara, transparente y accesible a sus potenciales usuarios (Muñoz Canãvate, 2003, p. 42; 2009: 476).

Una cuestión concomitante fundamental para favorecer el intercambio de la información de las administraciones públicas es la utilización de técnicas documentales y esquemas de metadatos, que se analizan a continuación.

\subsection{La utilización de técnicas documentales y esquemas de metadatos}

Autores como Chamorro (2006) consideran las nuevas tecnologías como la base fundamental de la Administración Pública, permitiendo el desarrollo de sus servicios y la incorporación de otros nuevos. Codina (1994) considera que un sistema de información documental debe especializarse en la explotación del conocimiento y señala la importancia de los modelos conceptuales en los sistemas, dado que facilitan la comunicación entre los equipos de personas implicadas en tareas comunes, disminuyendo la ambigüedad y posibilitando el uso de un lenguaje común entre los miembros del equipo para evitar malentendidos, especialmente dados a aparecer, paradójicamente, en todos los asuntos relacionados con el desarrollo de los sistemas.

Codina (1994) conecta el concepto de sistema con las tareas de gestión de la información, ya que propone un concepto de sistema que contenga conjuntos documentales o, en su defecto: a) proposiciones asociadas; b) un modelo general de un sistema de información documental, c) un modelo general de los procesos de transformación que tienen lugar en el interior del sistema; d) una proposición general sobre la documentación y e) dos proposiciones particulares, relativas al estatuto de la documentación como disciplina científica y a su relación con otras disciplinas, cuyo objeto de estudio también es la información en alguna de sus facetas. García Marco (2011, p. 3) también pone de manifiesto el papel que desempeña el diseño de sistemas en la eficacia y eficiencia de la recuperación de la información.

La utilización de un modelo de representación de la información, basado en descriptores y lenguajes documentales (Codina, 1994) y la utilización de metadatos Martínez Usero (2006, p. 118) también se consideran esenciales para garantizar el potencial de la Administración Pública, especialmente debido a la ingente cantidad de información generada para su uso.

Respecto a los lenguajes documentales, Currás (2005) plantea tres definiciones para los tesauros, su máxima expresión:

[...] lenguajes terminológicos normalizados, postcontrolados y postcoordinados, usados con fines clasificatorios dentro de las disciplinas documentario-informativas.

[...] lenguaje artificial, construido con una finalidad preconcebida, en el que sus elementos lingüísticos -términos- se hallan en relación simultánea y recíproca, incluyendo correlaciones sintácticas y semánticas.

[...] sistemas dinámicos y evolutivos, en los que los nodos -términos- que los componen establecen correlaciones sintácticas y semánticas en interrelación dialéctica consigo mismos y con el medio en que se encuentran. En consecuencia, son portadores de información $\mathrm{y}$, al mismo tiempo, sirven para su ordenación y comunicación.

Se considera muy importante la aportación de Currás al considerar que un tesauro no solo facilita la recuperación de la información, sino que es en sí mismo un portador de información.

Respecto a los metadatos, Martínez Usero (2006, p. 114) los define como "toda aquella información descriptiva sobre el contexto, calidad, condición o característica de un recurso que tiene la finalidad de facilitar su recuperación, autentificación, evaluación, preservación o interoperabilidad." Méndez Rodríguez (2006, p. 84) también define metadatos como información estructurada y descriptiva sobre los recursos electrónicos para mejorar, entre otras cosas, la recuperación de información. Finalmente, Bustelo Ruesta (2011, p. 131) define también a los metadatos como la información que nos permite clasificar los documentos en una estructura predefinida, limitar o expandir el acceso y uso, identificar las acciones de disposición o preservación previstas, o recoger los eventos que le han sucedido a un documento a lo largo del tiempo. La autora considera que para gestionar la información hay que implicarla en los demás sistemas utilizados o desarrollados por las organizaciones. Un ejemplo en concreto puede ser MIREG (Managing Information Resources for eGovernment), una aplicación creada para la normalización en el uso de metadatos en los gobiernos y parlamentos europeos y para el desarrollo de las herramientas de gestión de metadatos que contribuyen para esta actividad. Está basada en las recomendaciones de las administraciones públicas de los estados miembros, así como en otras de carácter internacional. 
De esta forma, se pretende producir un marco adaptado al tratamiento de la información pública con algunos extras como el control del vocabulario, el uso de ontologías y mapas tópicos, y las directrices sobre buenas prácticas. Bustelo Ruesta $(2011$, p. 131) considera, al hablar de documentos electrónicos, que la gestión de metadatos se convierte en la verdadera tarea de la gestión de la información, entendiendo por documento electrónico los ficheros que contienen no solo la información sino también los metadatos necesarios para gestionarlos.

Para desarrollar de forma más eficiente un tipo $\mathrm{u}$ otro de metadatos, se debe determinar el objetivo principal de su creación (descripción de materiales, conservación de estos o administración de tareas), así como el modelo de codificación, que puede ser centralizado en un grupo de personas que los generan todos, o descentralizado, de forma que existan varias personas o servicios responsables de su asignación en cada fase del proceso de workflow. Además, la organización debe contar con una persona responsable de la gestión completa del proceso.

En definitiva, en el seminario celebrado en 2009 por el Comité Europeo de Normalización (CEN), se reconocía que el descubrimiento y el acceso a los servicios de la administración electrónica es a menudo difícil, no solo por una simple ausencia de clasificación, de una correcta terminología o por descripciones inexistentes de los servicios, sino también por problemas de lenguaje o de otras habilidades semióticas en falta o mal gestionadas. Según Hernández (2009, p.73), en las conclusiones de dicho seminario se resumían los obstáculos para ampliar el acceso y la reutilización de los recursos de la administración electrónica en tres tópicos (figura 3).

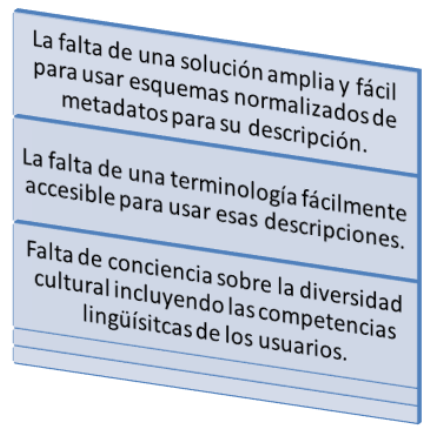

Figura 3. Obstáculos para ampliar el acceso y la reutilización de los recursos (Hernández, 2009, p. 73)

La importancia de la coexistencia entre sistemas informáticos ha crecido durante los últimos años. De igual forma, y como se ha visto anteriormente, en estos momentos se empieza a prestar mayor atención a la interoperabilidad de los datos, a la información y el conocimiento organizativo. En este caso, los metadatos pueden constituirse como una útil herramienta para asegurar el intercambio de informaciones y la integración de recursos muy heterogéneos entre sí. Un importante número de gobiernos en todo el mundo reconoce la necesidad de establecer estándares de metadatos como un ingrediente integral y fundamental de su marco de interoperabilidad (Hernández, 2009, p. 73).

Finalmente, para la implementación con éxito en la Administración Pública de todo lo citado con anterioridad se considera necesario llevar a cabo entre los usuarios un plan de Alfabetización Informacional.

\subsection{La importancia de la incorporación} de la alfabetización informacional

La ACRL (Association of College and Research Libraries, 2000) define la Alfabetización Informacional (en adelante en el texto Alfin) como

[...] un conjunto de habilidades que hacen que los individuos reconozcan cuándo se necesita la información y tengan la habilidad de localizar, evaluar y utilizar con eficacia la información necesaria.

En consecuencia, una persona con unos altos niveles de Alfin puede determinar el alcance de la información que necesita, acceder a ella, evaluar de forma crítica dicha información y sus fuentes, e incorporarla a su propia base de conocimientos. Asimismo, puede utilizarla de forma eficaz y conseguir lograr sus objetivos. Además, un usuario Alfin debe poder comprender las cuestiones económicas, legales y sociales que rodean al uso de la información, y acceder y utilizar la información de forma ética y legal (Association of College and Research Libraries, 2000).

Para Bernhard (2002, p. 421), la Alfin consiste en un conjunto de aptitudes referidas al uso y dominio de la información en cualquiera de las formas en que se presente, así como de las tecnologías que dan acceso a esta información, capacidades, conocimientos y actitudes relacionadas con la identificación de las necesidades de información, el conocimiento de las fuentes de información, la elaboración de estrategias de búsqueda y localización de la información, la evaluación de la información encontrada, y su explotación, reformulación y comunicación.

Para Ponjuan (2007, p. 37), una persona ha desarrollado una cultura informacional cuando tiene las habilidades de definir tareas, crear sus propias estrategias de búsqueda de información, localizando y accediendo para poder hacer 
uso de ella, además de su análisis y evaluación. En la misma línea, Angulo (2003) hace una llamada de atención sobre la importancia de la formación para gestionar recursos y, así, poder obtener una recuperación de la información eficiente, ya que el desarrollo y el uso intensivo de las tecnologías de la información ha favorecido una diversificación y un incremento sin precedentes de la información disponible, tanto en formato impreso como en formato electrónico. Sin embargo, este incremento no ha ido acompañado de mecanismos de control de calidad ni de elementos para asegurar una mayor precisión en la representación y la organización de la información, lo que crea el riesgo de que la información valiosa se pierda entre la información obsoleta, redundante, imprecisa, tendenciosa o mal estructurada. En definitiva, la alfabetización informacional y la formación implementada en la gestión de recursos nos van a llevar a unas mejores condiciones de incrementar el proceso de recuperación de la información (figura 4).

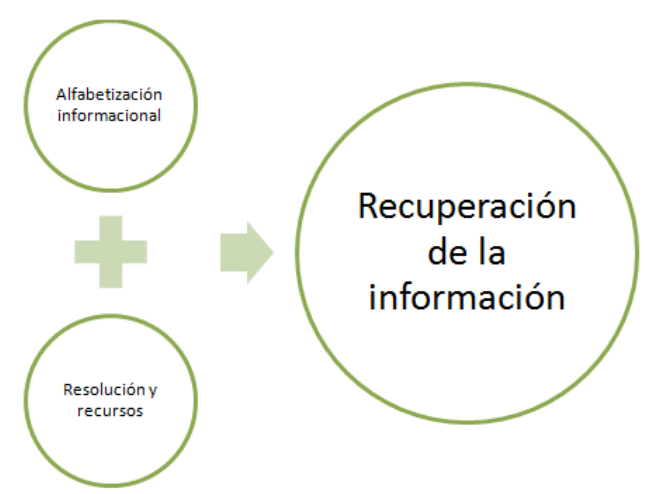

Figura 4. Relación entre Alfin, recursos y recuperación de información

La misma idea de la formación ha sido seguida en otros países, ya que la expresión alfabetización informacional corresponde a la traducción literal de information literacy, un término utilizado en los países de habla inglesa para referirse a una diversidad de competencias que van desde la capacidad de usar la información para resolver problemas, diseñar y aplicar estrategias eficientes de búsqueda de información, hasta operar con las herramientas prácticas y conceptuales de las tecnologías de la información para ubicarla, accederla, procesarla, diseminarla y convertirla en conocimiento.

Según Ortoll (2003, p. 8), la competencia informacional va a proporcionar a la persona mecanismos para saber identificar una necesidad de información, el tipo de información que puede resolver dicha necesidad y los recursos más apropiados para proporcionar dicha información. A su vez, estos mecanismos son esenciales para la identificación de conocimiento relevante y de las fuentes para su localización. La misma autora identifica, además, el comportamiento informacional con el conocimiento para realizar las búsquedas que van a permitir a la persona, además de la extracción de la información, evaluar su coste. Una vez alcanzada la alfabetización informacional, como consecuencia tenemos un conocimiento que nos aporta la capacidad de aplicar los mecanismos necesarios para organizar y estructurar la información de manera que se facilite el trabajo y el poder aplicar las técnicas adquiridas con el fin de que sea fácilmente recuperable. Del mismo modo, adquirir la habilidad de adjudicar palabras clave que identifican el contenido de la información que se resume. Dichos elementos se presentan como esenciales en el proceso de explicitar, estructurar y almacenar conocimiento. Asimismo, en el proceso de compartir y transmitir conocimiento, los mecanismos, herramientas, normativas y estrategias para comunicar ideas de forma efectiva, así como para integrar información de distintas fuentes, con el fin de crear sinergias entre ellas, se encuentran implicados.

Finalmente, las aportaciones de la pedagogía informacional aplicadas a las estrategias de evaluación, análisis, síntesis, selección o filtro de información, identificando las diferencias entre la información que proviene de distintos recursos, y planteando formas para aplicarla a la resolución de un problema, son de igual modo elementos clave para la reutilización y la renovación del conocimiento existente.

\section{Conclusiones}

En este artículo se han presentado propuestas para facilitar la disminución de los factores que inhibien y dificultan la recuperación de la información en la Administración Pública española. Y se ha lamado la atención sobre su relación con las Ciencias de la Documentación y también sobre la importancia que tiene la gestión de la información, la organización de la información, para la Administración Pública española.

Se invita por tanto con este trabajo a continuar analizando la gestión de la información en la Administración Pública española desde la perspectiva de la recuperación de la información, a reflexionar sobre su importancia; y observar si los resultados propuestos para facilitar la disminución de los factores inhibidores de la recuperación de la información en la Administración Pública van siendo implementados; para, finalmente, conocer, si una vez implementados, han 
sido suficientes para reducir los factores inhibidores o por el contrario se considera necesario investigar más.

Como gestores de la información estamos obligados a intentar que se aplique la gestión informacional y funcione, para que de esa forma, la información, pueda ser utilizada como herramienta para que organizaciones y usuarios puedan cumplir con sus objetivos y así nosotros ver cumplir también el nuestro.

\section{Notas}

(1) Según la norma internacional ISO 15489-2, Records Management es la "disciplina encargada del control eficiente y sistemático de la creación, recepción, mantenimiento, uso y eliminación de récords, incluyendo el proceso de captura y mantenimiento de las evidencias e informaciones acerca de actividades de negocio y transacciones en la forma de récords" (ISO-UNE, 2006: 8).

\section{Referencias}

Alcaide Muñoz, L. (2012). E-Gobierno e información financiera pública. Estudio metaanalítico de los determinantes de la divulgación y sus efectos moradores. Granada: Departamento de Economía Financiera y Contabilidad, Universidad de Granada. Tesis doctoral.

Angulo Marcial, N. (2003). Normas de competencia en información. http://www2.ub.es/bid/consulta_articulos.ph p?fichero=11angul2.htm (2013-01-15).

Bernhard, P. (2002). La formación en el uso de la información: una ventaja en la enseñanza superior, situación actual. // Anales de Documentación. 5, 409-435.

Bustelo Ruesta, C. (2011). Los grandes temas relacionados con la gestión de documentos: desafíos y oportunidades. // El profesional de la información. 20:2, 129-133.

Chamorro, M. (2006) Líneas estratégicas, Ministerio de Fomento. [en línea]. Tecnimap, Sevilla, 2006. [ref. de 06 de Noviembre de 2011] www.administracionelectronica. gob.es/recursos/pae_000002317.pdf

Codina, L. (1994). La naturaleza de la recuperación de la de información: implicaciones para el diseño de sistemas de información documentales. Oviedo: Universidad de Oviedo.

Costa, L.; Silva, A.; Ramalho, F.(2010) Para além dos estudos de uso da informaçao arquivística: a questão da accesi-bilidade. // Ci. Inf. 9:2, 129-143.

Cruz Gil, M. C. (2012). Vías de optimización de los sistemas de recuperación de la información. Ministerio de Fomento. TFM. Máster SIC. Universitat Oberta de Catalunya.

Currás, E. (2005). Ontologías, taxonomía y tesauros: manual de construcción y uso. $3^{\mathrm{a}}$ ed., actual. y ampl. Gijón: Trea, 2005.

García Alsina, M. (2008). Metodología para la implantación de la gestión del conocimiento a partir de técnicas documentales. // Scire. Representación y organización del conocimiento. 14:1, 43-61.

García Marco, F. J. (2008). Gestión de la información y del conocimiento en las organizaciones: perspectivas y es- tudios de caso. // Scire: Representación y organización del conocimiento. 14:1, 9-14.

García Marco, F. J. (2011). Psicología y sociología de la información: una necesidad práctica y teórica. // El profesional de la información, 20:1, 5-9.

García, O. (2010). Modelo teórico para el desarrollo de aprendizaje organizacional en el contexto de los nuevos paradigmas de la sociedad de la información. // Revista Digital sociedad de la información. 19, 1-11.

González Lorca, J. (2006). Sistemas workflow. Funcionamiento y metodología de implantación. Gijón: Ediciones Trea.

Hernández, T.; et al. (2009). El uso de metadatos en la administración electrónica española: los retos de la interoperabilidad. // Revista Española de Documentación Científica. 32:4, 67-91.

Martín-Pozuelo; M. P. (2012). El observatorio de prospectiva archivística y sociedad: modelo de análisis para el estudio de la construcción social del futuro de al archivística. // Novas dimensoes da pesquisa e do ensino da Arquivologia no Brasil / organizaçao de Anna Carla Almeida Mariz, José María Jardim, Sergio Conde de Albite Silva. Rio de Janeiro: Móbile: Associaçao dos Arquivistas do Estado do Rio de Janeiro, 2012.

Martínez Usero, J.A. (2006). El uso de metadatos para mejorar la interoperabilidad del conocimiento en los servicios de administración electrónica. // El profesional de la información. 15:2. pp. 114-126. ISSN 1386-6710.

Méndez Rodríguez, E.M. (2006). Dublin Core, metadatos y vocabularios. // El profesional de la Información. 15:2, 84-86.

Muñoz Cañavate, A. (2003). La administración general de estado en internet: un estudio sobre la VI legislatura. Badajoz: Instituto de Ciencias de la Educación, Facultad de Biblioteconomía y Documentación.

Muñoz Cañavate, A. (2009). Bases de datos de libre acceso difundidas por la Administración General del Estado (L. Fernando Ramos-Simón). // El profesional de la información. 18:4, 475-477.

Ortoll, E. (2003). Gestión del conocimiento y competencia informacional en el puesto de trabajo. http://www.uoc. edu/dt/20343/index.html (2012-01-14).

Pastor Sánchez, J. A. (2014). Aspectos prácticos para proyectos de datos abiertos en las administraciones públicas. // Anuario ThinkEPI. 8, 313-317.

Ponjuan, G. (2007). Gestión de información: dimensiones e implementación para el éxito organizacional. Gijón: Edicio-nes Trea.

Ruthven, I. (2008). Interactive information retrieval. http://www.cis.strath.ac.uk/cis/research/publications/pap ers/strath_cis_publication_2230.pdf (2013-02-02).

Solana, P., Alonso, M., Pérez, D. (2007). Análisis y modelado con redes de workflow del proceso de tratamiento de experiencias operativas. dialnet.unirioja.es/descarga/arti culo/2487713.pdf (2014-04.15)

Wilson, T.D. (1999). Models in information behavior research. http://informationr.net/tdw/publ/papers/1999J Doc.html (2013-01-23).

Enviado: 2015-07-28. Segunda versión: 2015-05-05. Aceptado: $2015-06-26$. 